\title{
THE BOLOMETRIC LUMINOSITIES OF STARS
}

\author{
R. C. BLESS AND J. W. PERCIVAL \\ University of Wisconsin - Madison, 53706, USA
}

\begin{abstract}
The total energy emitted by a star is one of its basic parameters. It is also one of the most difficult to determine, requiring space-based as well as ground-based measurements of the absolute flux from stars. We review the current status of these observations in the ultraviolet, visible, and near infrared, and estimate the uncertainties of the absolute flux measurements in these spectral regions. We compare recent determinations of the bolometric luminosity of early-type stars with the first (mostly) empirical results of 20 years ago. We close with a few comments on possible improvements of these measurements in the future.
\end{abstract}

\section{Introduction}

The link between stellar atmospheric models (theory) and real stars (observations) has been through their effective temperatures and bolometric luminosities. It is only in the last 20 years or so, with observations made from space along with techniques for measuring angular diameters, that these latter two quantities could be directly determined by observations for stars other than the sun. Previous speakers have given the basic relationships. Let us just remind you that the effective temperature of a star is proportional to the square root of its angular diameter and the fourth root of the total flux measured at the Earth. If necessary, this flux must be corrected for interstellar extinction, which of course introduces uncertainty. Except for the use of models in finding the limb-darkened angular diameter, the star's effective temperature can be found completely empirically (at least in principle), subject to the errors in the measured flux and angular diameter. Neither of these, however, enters strongly into its determination.

If, in addition, the parallax of the star is well-determined, then its radius follows directly from its angular diameter. With the effective temperature 
and radius (assuming, of course, that the star has a well-defined radius), the luminosity of the star can be calculated and a fundamental HR diagram (luminosity vs. effective temperature) can be plotted. Thus the physics involved is quite straightforward; getting the relevant data is much less so. Before describing the latter, let us quickly remind you in which wavelength regions most of the flux emerges for the various spectral types.

We integrated the emergent flux from several Kurucz (1992) models at a variety of effective temperatures over several wavelength bands, corresponding roughly to the extreme ultraviolet, the $300 \AA$ between Ly- $\alpha$ and the Lyman continuum (the FUV), the conventional UV, the visual and two bands in the infrared. The fractional fluxes in these bands, plotted as a function of effective temperature, are shown in Figure 1. Note that the EUV region becomes significant only for stars having effective temperatures greater than about $30,000 \mathrm{~K}$, while even the coolest stars radiate little in the $4-10 \mu$ region. The $1-4 \mu$ region, however, is about as important as the visual region for the sun and cooler stars. We must keep this in mind when assessing the flux accuracy possible for a given spectral type.

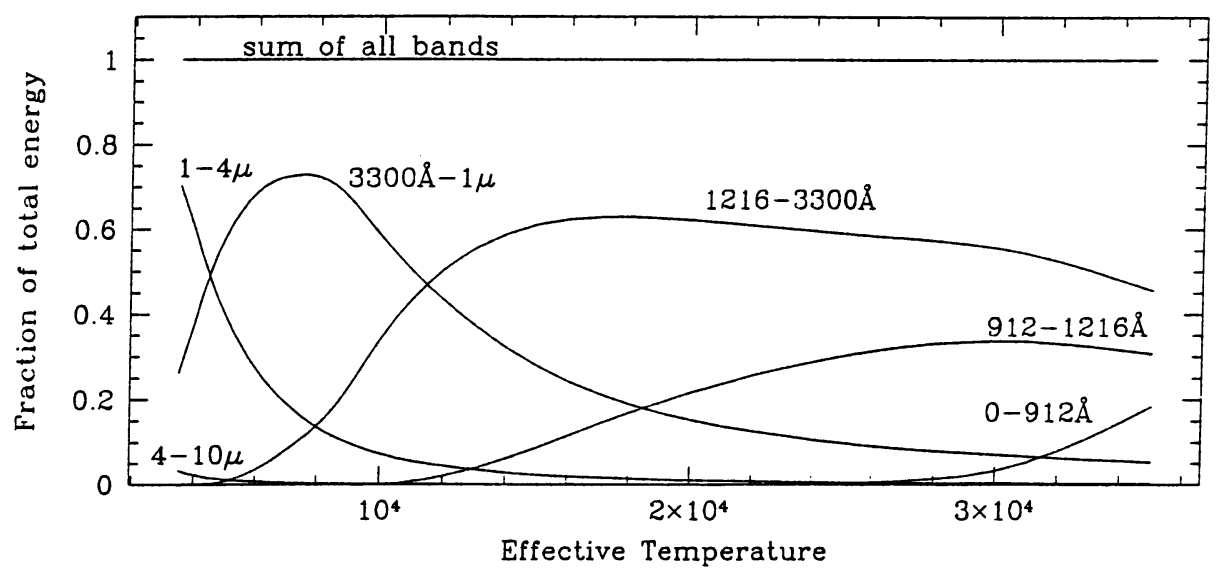

Figure 1. Kurucz models by wavelength region

Time does not allow a discussion of all absolute calibration work and for the most part in this review we will concentrate on the most recent efforts.

\section{The Ultraviolet Spectral Region}

We will begin this discussion with the conventional ultraviolet because it was in this spectral region that the white dwarf model calibration system, now the standard, was first developed. With the capability of observing stars from above the earth's atmosphere about 40 years ago came the chal- 
lenge of absolute calibration in the ultraviolet. Kruk (1997) briefly describes some of the earlier calibration efforts and their difficulties that led to the use of white dwarf model atmospheres for calibration purposes.

Though the use of hot DA white dwarf model atmospheres for absolute flux calibrations was apparently first suggested by Greenstein and Oke in 1979 , it has been only in the last several years that this idea has been put into wide practice. One chooses the appropriate model effective temperature and surface gravity from the best fit to the profiles of several hydrogen lines in each of the white dwarf calibration stars. The model fluxes are then normalized to the visual magnitude of the white dwarf being modeled. An instrument and telescope, for example, the Faint Object Spectrograph on the Hubble Space Telescope, can then be calibrated by comparing its response to the predicted white dwarf model flux.

White dwarf models are attractive for several reasons. The hot ( $>$ $20,000 \mathrm{~K}) \mathrm{DA}$ white dwarfs have nearly pure hydrogen, plane-parallel, nonconvective, essentially LTE atmospheres and have no winds. Apart from the hydrogen lines, their spectra are very nearly featureless. Above temperatures of about $30,000 \mathrm{~K}$, relative fluxes are nearly independent of gravity. At $50,000 \mathrm{~K}$, an effective temperature error of $1000 \mathrm{~K}$ leads to errors of only $0.5 \%$ in the long wavelength portion of the Balmer continuum, increasing to about $1 \%$ at Ly- $\alpha$. Internal accuracies of $1.5 \%$ or less should be attainable over the optical and UV spectral regions. The models do have some deficiencies of course, e.g. the lack of a rigorous Stark broadening theory. Nevertheless, model atmospheres of hot DA white dwarfs represent an attractive alternative to laboratory calibrations, so attractive, in fact, that today the calibrations of both the IUE archive and HST data are based on DA white dwarfs.

The fundamental standards used by Bohlin (1996) to define the HST white dwarf scale are an average of the pure hydrogen LTE models calculated by D. Finley using D. Koester's code for HZ 43, GD 71, GD 153, and G191-B2B of which the last is by far the brightest. Hubeny (1996) computed non-LTE atmospheres for the same temperatures and surface gravities as Koester's models. The agreement with all but G191-B2B is very good, but for the latter, Hubeny's fluxes are about $0.5 \%$ lower shortward of $3500 \AA$ and up to $3.5 \%$ higher at $2.5 \mu$. This latter difference may be a reflection of the poorer model accuracy in spectral regions where little flux is emitted. Lanz et al. (1996) computed a non-LTE model of G191-B2B blanketed by about 9,000,000 lines. With an effective temperature of $55,200 \pm 1000 \mathrm{~K}$ it agrees with the Koester model within $1 \%$ from $20 \mu$ to about $1700 \AA$. From 1700-1000 $\AA$ many absorption lines depress the spectrum by a few per cent.

As attractive as this approach may be, to have such an important stellar datum based on theory is not an acceptable state of affairs. Consequently, 
the calibration of the Hopkins Ultraviolet Telescope on ASTRO just described by Jeff Kruk is very important (Kruk et al. 1997). He and his colleagues are to be congratulated for carrying out this program. Their work confirms the white dwarf scale over the spectral region from $912-1840 \AA$ to within the error of their laboratory calibration, roughly $5 \%$. Bohlin feels that the white dwarf models give calibrations good to $3 \%$ from $1150 \AA$ through the optical, a number in line with the Hopkins calibration work.

Now, as just noted, the atmosphere of G191-B2B contains some metals. However, the Hopkins group showed that within their error limit, the spectrum of this white dwarf is indistinguishable from that of a pure hydrogen atmosphere at HUT resolution in the FUV. It appears, therefore, that despite its metal content, G191-B2B is a satisfactory calibration star in the FUV and UV, at least at the $5 \%$ level, and that the calibration does not depend strongly on the white dwarf model employed.

These numbers suggest that if you are careful and lucky, it should be possible to produce spectrophotometry in the UV to the Lyman limit that is accurate to about $5 \%$. Part of the luck just mentioned is the correction for interstellar extinction. If the (B-V) color excess is in error by just 0.01 magnitudes, the extinction correction at $1500 \AA$ and $1000 \AA$ could be in error by 5 and $10 \%$, respectively. For stars that radiate much of their energy in the UV, this could be the dominant source of error. Buss et al. (1995) in analyzing observations of about a dozen OB stars obtained by HUT from $912-1840 \AA$, estimate their calibration uncertainty to be about $5 \%$, but extinction uncertainties to be as large as $15 \%$

Both the final IUE archive and HST spectra are being calibrated on the white dwarf scale. Though the spectra of the same star observed by IUE and HST will have about the same shape, they will not have the same absolute flux level. This results from two factors: first, the IUE calibration takes the effective temperature of its standard, the white dwarf, G191-B2B, to be $58,000 \mathrm{~K}$, which is $3,000 \mathrm{~K}$ lower than the temperature taken for this star in the HST calibration. This results in a maximum difference of about $1.3 \%$. Secondly, the HST zero point is based on the measured V magnitudes of Landolt (1992). The IUE calibration, however, takes as its zero point OAO-2 fluxes of the brightest IUE standards in the spectral region of 2100$2300 \AA$, chosen because there the OAO- 2 and TD- 1 observations agree best. The argument for normalization in the UV rather than in the visual is that it avoids an extrapolation from the visual to the UV and thereby minimizes the effects of errors in both the model and the observations which otherwise might be introduced. This difference in zero points amounts to about $5 \%$. Thus, the HST fluxes are up to $6 \%$ brighter than the new IUE fluxes, independent of wavelength. The Hopkins laboratory calibration gives a normalization that is about $4 \%$ brighter than that of IUE spectra on the 
white dwarf scale. This difference is in the same sense and nearly the same amount as found by Bohlin and suggests that the HST normalization is to be preferred over the IUE scale.

\section{The Visual and Infrared Spectral Regions}

We turn next to the calibration of the visual region of the spectrum on which work has been done for the longest period of time and which is probably in reasonably good shape. Vega has traditionally been taken as the standard star for this region of the spectrum. Hayes (1985) has reviewed the absolute calibration of Vega and recommends a weighted mean of six determinations from $3300-10,500 \AA$. He feels that the internal error of this average is good to $1-1.5 \%$, that is, about as accurate spectrophotometry as can be done. The measurements of Hayes and Latham (1975) do not differ significantly from this mean curve and so some authors still use these observations. In the optical, the white dwarf scale agrees with the Hayes Vega calibration to about $3 \%$ and so gives a somewhat poorer calibration.

As we have just heard, Dr. Megessier has reviewed the various determinations of the absolute flux from Vega and recommends that the value of $F_{5556 \AA}=3.46 \times 10^{-9} \mathrm{ergs} \mathrm{cm} \mathrm{cm}^{-2} \mathrm{~s}^{-1} \AA^{-1}$. This differs by about $0.5 \%$ from the value recommended by Hayes, well within the error of about $1.5 \%$ he quotes. Realistically, the absolute visual flux of Vega is probably known about as well as can be at the present time.

An observer using these data must compare spectrophotometry of the program star with that of Vega or some other well-observed standard star, as well as determine accurately the apparent magnitude of the star in a well-defined bandpass. With great care, spectrophotometry can be done to $1-2 \%$ and the apparent magnitude to perhaps $0.5 \%$. Adding all these errors (those of the standard star as well as the program star) in quadrature gives a mean error for program stars of about $2.5 \%$ on the Vega scale or about $3.5 \%$ on the white dwarf scale. This is probably the best that can be done now and many observations will not achieve this accuracy. Hall (1996) gives a compilation of 9 major sources of absolute visual spectrophotometry for which the average accuracy is $4 \%$, probably a realistic figure.

Ground-based absolute IR observations, for example in the broad $\mathrm{J}, \mathrm{H}$, $\mathrm{K}$, and $\mathrm{L}$ bands, are difficult to make accurately. In this spectral region, absorption by the earth's atmosphere can change rapidly during a night. To minimize these effects, some observers use much narrower filters than those of the Johnson system. Care is required in relating such observations to those in the Johnson system, however, where the shape of the bandpass, at the temperature at which they are actually used, must be determined accurately. 
Just as in the visible, Vega has been used as a standard star in the IR. A model atmosphere fit to the visual is assumed to represent Vega in the infrared and provide the absolute calibration. Some argue, however, that in the near IR Vega is systematically brighter than the model by a small amount. That is, the model atmosphere does not represent the real Vega. Megessier (1995) emphasizes this point and derives a near IR flux calibration based on solar analog stars or on blackbody furnaces, but not on model atmospheres. In doing this she finds consistent results among various authors and estimates errors of about $2 \%$ in the absolute calibration and $2-3 \%$ in the relative photometry. Thus, the IR calibration from $1-4 \mu$ is thought to be about as accurate as the absolute calibration in the visual.

\section{The Extreme UV Region}

We will turn finally to the data produced by the EUV Explorer. Three spectrometers, each with its own detector, cover the spectral range from $70-760 \AA$ with resolution of about 200 . The Berkeley group, in preparing an EUV spectral atlas of many stellar objects, is establishing a final instrumental calibration. The contamination of the long wavelength spectra by overlapping orders from the shorter wavelengths is just one of several problems that must be addressed. Before launch, a sensitivity model for the instrument that was no better than about $20 \%$ was produced. After launch, observations of white dwarfs, especially $\mathrm{HZ} 43$, were combined with the lab calibrations to reduce the errors to about $10 \%$, except over the $600-700 \AA$ region where the calibration is poorer. This is thought to be representative of the current calibration state of the instrument.

Figure 2 shows the pure hydrogen, LTE model spectra of the four fundamental HST calibration white dwarfs and their spectra observed by EUVE, HUT and HST from 100-10,000 $\AA$. That they fit together nicely should be no surprise since they are all based on the same calibration. Probably the most striking feature of the observations is that of G191-B2B which shows the effect of a large amount of interstellar absorption by hydrogen and helium in the FUV. Though the shape of this absorption is well known from theory, the total amount is less certain. Unless this absorption can be accurately predicted independently, it is hard to see how this star can be a reliable calibration object in the EUV. This is particularly true since Lanz et al. (1996) point out that the He I edge in G191-B2B may be partly stellar or circumstellar rather than entirely interstellar. From that point of view, GD 71 is much to be preferred, but unfortunately it is faint in the EUV region. Until a detailed account of the EUVE calibration procedures and results are published, it will be difficult to assess the accuracy of its calibration. 

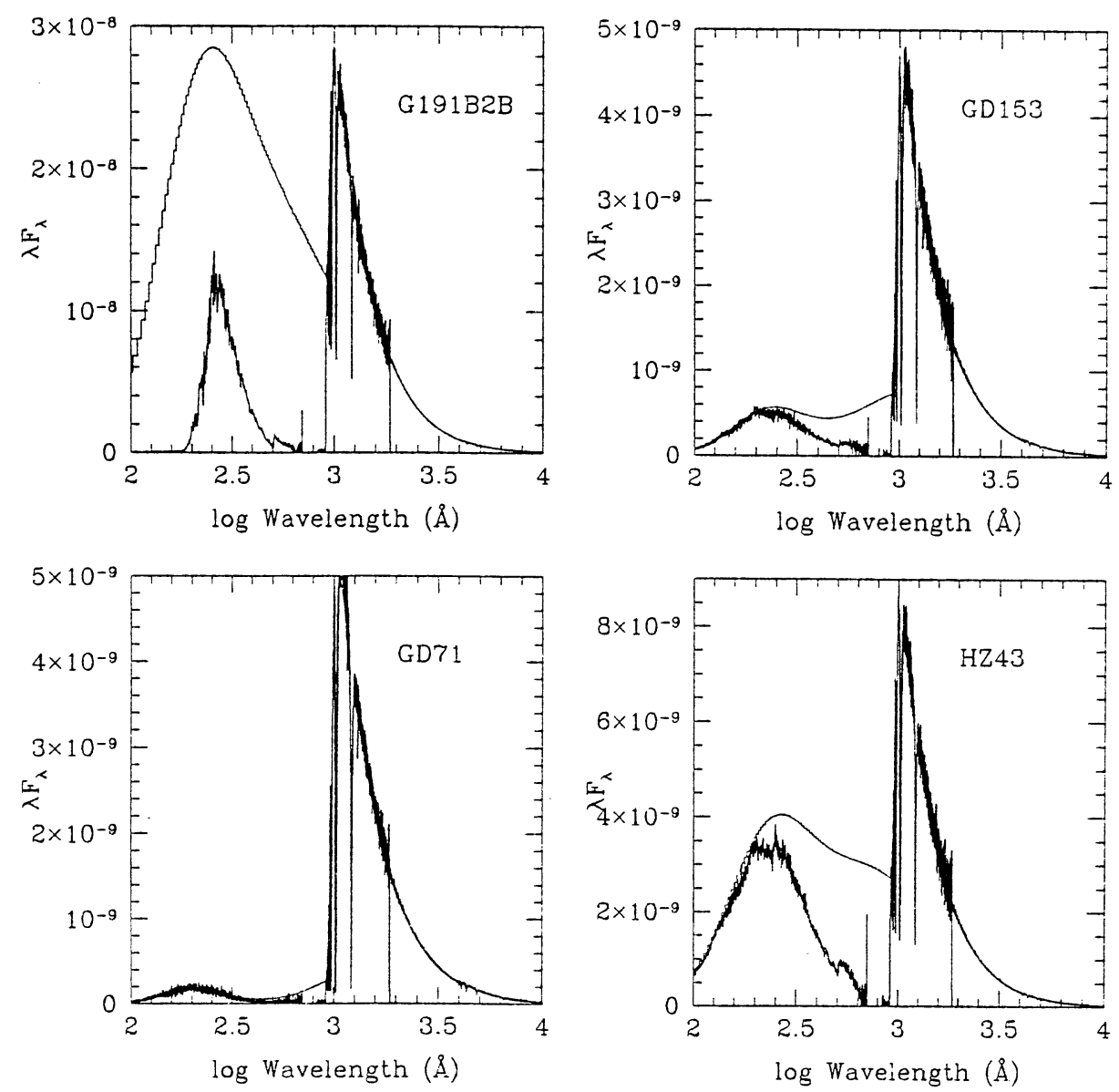

Figure 2. White dwarf models compared to observations

In summary, it appears that with great care and accurate extinction corrections, program stars can be absolutely calibrated to about $5 \%$ from the Lyman limit to the visible and to about $3-4 \%$ from the visible to $3 \mu$.

\section{Comparison With Previous Data}

About 20 years ago Hanbury Brown and colleagues (1974) completed measurements of the angular diameters of 32 mostly early-type stars with the Narrabri interferometer. At the same time, the Wisconsin group provided the ultraviolet fluxes from these stars measured with the OAO-2 satellite observatory. Sonce these stars radiate most of their energy in the UV and visual, the derived effective temperatures and bolometric corrections were, in large part, empirically determined. 
It is of interest to see by how much the original OAO fluxes differ from those that would be determined on the current white dwarf scale. The differences in the UV flux are systematic and surprisingly small because the two calibration changes (OAO to IUE and IUE to HST) cancel each other to some extent. Since the old and new visual calibrations are essentially the same, these results mean that for the most part, effective temperatures of these stars based on the new white dwarf scale will differ by no more than about $1 \%$ from those reported 20 years ago, with the cooler stars becoming slightly warmer, and the hotter stars slightly cooler. We are presently rederiving fluxes and effective temperatures of these and other stars in detail using the new calibrations.

\section{The Future}

Can the measurements of stellar flux described here be significantly improved in the future? We are not aware of any extensive programs of fundamental absolute calibration now underway or being planned, though we have not made a detailed survey. This is regrettable, but understandable, given the difficulties of this work, not the least of which is acquiring the funding for it.

Absolute calibration in the EUV region is probably the least well known, not surprising given the experimental difficulties in this spectral region. Whether the recent flight of ORPHEUS will improve matters is not known at the present time. EUV calibration is important not only in helping to establish the white dwarf scale in the UV to the IR, but also for understanding the EUV flux from ordinary hot stars. Cassinelli et al. (1995) found that $\epsilon$ CMa radiates about 30 times as much energy in the EUV than predicted by an appropriate $\mathrm{B} 1$ giant model. This result indicates that $\epsilon \mathrm{CMa}$ is the dominant source of ionizing flux for interstellar gas within a few parsecs of the sun, a completely unexpected result. This suggest that not only our knowledge of stellar models for hot stars, but also of the ionization equilibrium in the interstellar medium, is incomplete. DA white dwarfs used for absolute calibration may well have simpler atmospheres than B-type giants. Nevertheless, it is a bit worrisome that this discrepancy between model and star exists in the spectral region, the EUV, that is largely calibrated by a model.

A relatively simple but quite useful near IR program would be absolute photometry of the white dwarf calibration stars. Since this could be done from the ground, the opportunity exists to establish by observation the validity of the white dwarf calibration in this spectral region rather than relying on the model atmospheres, which, as noted earlier, may not be as accurate in the IR as in the UV or visible. 
The $2.5-45 \mu$ spectrometer $(R=1000)$ on ISO should be useful for absolute calibration purposes. Its calibration is based primarily on observations of mostly late-type standard stars, not white dwarfs. In turn these depend on the careful absolute calibration work given by Cohen et al. (1996 and references therein) in several papers. They estimate that from $1.2-35 \mu$ the uncertainty in their calibration is about $3 \%$. They feel, however, that the case for a small near IR excess in Vega is not convincing. This should be kept in mind when using ISO data.

A light source in the sky, but calibrated on the ground, would be desirable. It might be possible to mount such a calibration source on the space station, assuming it ever gets built. In principle, lamps could be calibrated on the ground and gotten into use on relatively short time scales, and similarly returned to ground for recalibration. Many technical problems would have to be overcome, for example, tracking the source from a moving satellite. However, it would also provide some useful science for the space station to do.

ESTEC is developing a promising new detector based on superconducting tunnel junctions. Photoabsorption in a niobium junction, cooled to $0.3 \mathrm{~K}$, produces hundreds of free charge carriers for each incident event with a resolution of $100 \AA$ or less. These are detected when they quantum mechanically tunnel through an insulating barrier. Such a detector is a complete low resolution spectrometer having a small dark noise and no read-out noise, with high sensitivity from the UV to the near IR. Eventually, this sort of detector might be accurately calibrated in the laboratory and provide useful checks on the assumed calibrations.

Finally, a brief comment concerning fluxes for stars with measured angular diameters. As of now, angular diameters have been measured only for bright stars. Mid- to late-spectral type stars radiate most of their flux in the visual and infrared which can be measured from the ground. For early-type stars the UV is important, but HST cannot measure the bright stars for which there are angular diameters. One must hope that IUE observations made in the fast trail mode exist for the stars of interest. It may well turn out that some of the old observations made by OAO-2 and other satellites might have a rebirth of usefulness in this regard. We are looking into this possibility.

In summary, a fundamental change in the absolute calibration of stellar fluxes has been taking place over the last few years. It is less and less of an experimental enterprise and increasingly becoming one in which a computer is the primary piece of equipment. Model makers have become the calibrators. White dwarf model atmospheres do indeed seem to be reasonably successful as calibrating objects over much of their spectra and can be computed to seductively high internal precisions. It is important to 
remember, however, that the models are deemed successful in the visual and ultraviolet BECAUSE they are constrained by laboratory calibrations and observations. There appears to be less such constraint in the EUV region, so results from that spectral regions should be carefully evaluated. In general, caution should be exercised in accepting model results uncritically. In particular, we hope that independent calibration work does not disappear entirely, but will be continued, especially at the two ends of the spectrum-the IR and the EUV.

\section{Acknowledgements}

We are grateful to M. Abbott, R. Bohlin, J. Cassinelli, and J. Kruk for many helpful discussions, and to the Berkeley EUVE group (Craig, et al., 1996) for providing EUVE spectra prior to publication. We also thank I. Hubeny for the emergent flux over the whole spectrum of the Lanz et al. model of G191-B2B.

\section{References}

Bohlin, R.C. 1996, AJ, 111, 1743.

Brown, R.H., Davis, J., \& Allen, L.R. 1974, MNRAS, 167, 121.

Buss, R.H., Kruk, J.W. \& Ferguson, H.C. 1995, ApJ, 454, L55.

Cassinelli, J.P. et al. 1995, ApJ 438, 932.

Cohen, M., Witteborn, F.C., Bregman, J.D., Wooden, D.H., Salama, A., \& Metcalfe, L. 1996, AJ, 112, 241.

Craig, N., Abbott, M., Finley, D., Jessop, H., Howell, S., Mathioudakis, M.,

Sommers, J. Vallerga, J., \& Malina, R. 1996, private communication.

Hall, J.C. 1996, PASP, 108, 313.

Hayes, D.S. 1985, in Calibration of Fundamental Stellar Quantities,

Proc. IAU Symposium No. 111, eds. D.S. Hayes, L.E. Pasinetti,

\& A.G. Davis Phillip (Reidel: Dordrecht), p. 225.

Hayes, D.S. \& Latham, D.W. 1975, ApJ, 197, 593.

Hubeny, I., see Bohlin, 1996.

Greenstein, J.L. \& Oke, J.B. 1979, ApJ, 229, L141.

Kruk, J.W. 1997, these proceedings.

Kruk, J.W., Kimble, R.A., Buss, R.H., Davidsen, A.F., Durrance, S.T.,

Finley, D.S., Holberg, J.B., \& Kriss, G.A. 1997, ApJ 482, in press.

Kurucz, R.L. 1992, Smithsonian Astrophysical Observatory, CD-ROM No.

Landolt, A. 1992, AJ, 104, 340.

Lanz, T., Barstow, M.A., Hubeny, I., \& Holberg, J.B. 1996, ApJ, 473, 1089.

Megessier, C. 1995, A\&A, 296, 771.

\section{Discussion of this paper appears at the end of these Proceedings.}

